\title{
Reliability and validity of the Athens Insomnia Scale in chronic pain patients
}

\author{
Kiyoka Enomoto ${ }^{1-3}$ \\ Tomonori Adachi ${ }^{2-4}$ \\ Keiko Yamada ${ }^{5}$ \\ Daisuke Inoue ${ }^{2,6}$ \\ Miho Nakanishi ${ }^{7}$ \\ Tomohiko Nishigami ${ }^{2,8}$ \\ Masahiko Shibata ${ }^{1,2}$ \\ 'Department of Pain Medicine, \\ Osaka University Graduate School \\ of Medicine, Suita, Japan; ${ }^{2}$ Center \\ for Pain Management, Osaka \\ University Hospital, Suita, Japan; \\ ${ }^{3}$ Department of Anesthesiology, \\ Interdisciplinary Pain Management \\ Center, Shiga University of Medical \\ Science Hospital, Otsu, Japan; ${ }^{4}$ Japan \\ Society for the Promotion of Science \\ (JSPS), Tokyo, Japan; ${ }^{5}$ Public Health, \\ Department of Social Medicine, \\ Osaka University Graduate School of \\ Medicine, Suita, Japan; ${ }^{6}$ Department \\ of Occupational Therapy, Osaka \\ College of Rehabilitation, Osaka, \\ Japan; ${ }^{7}$ Department of Anesthesiology, \\ Shiga University of Medical Science, \\ Otsu, Japan; ${ }^{8}$ Department of Nursing \\ and Physical Therapy, Konan Woman's \\ University, Kobe, Japan
}

Correspondence: Kiyoka Enomoto Department of Pain Medicine, Graduate School of Human Sciences, Osaka University, 2-2 Yamadaoka, Suita, Osaka 565-087I, Japan

Tel +81668793745

Fax +8I 668793495

Email k.enomoto@pain.med.osaka-u.ac.jp
This article was published in the following Dove Press journal: Journal of Pain Research

Purpose: To confirm the psychometric properties of the Athens Insomnia Scale (AIS) among Japanese chronic pain patients.

Patients and methods: In total, 144 outpatients were asked to complete questionnaires comprising the AIS and other study measures. According to the original article, the AIS has 2 versions: the AIS-8 (full version) and the AIS-5 (brief version). To validate the AIS- 8 and AIS- 5 among chronic pain patients, we confirmed: 1) factor structure by confirmatory factor analysis; 2) internal consistency by Cronbach's $\alpha$; 3) test-retest reliability using with interclass correlation coefficients; 4) known-group validity; 5) concurrent validity; and 6) cut-off values by receiver operating characteristic analysis. In addition, semi-structured interviews were conducted to assess the participants' sleep disturbance. If the participants had any sleep complaints, including difficulty in initiating sleep, difficulty in maintaining sleep, and early morning awakening, they were defined as insomnia symptoms.

Results: A 2-factor model of the AIS-8 and 1-factor model of the AIS-5 demonstrated good fit. The AIS had adequate internal consistency and test-retest reliability. Patients with insomnia had a higher AIS score than those without insomnia. The sleep disturbance measured by the AIS was positively associated with pain intensity, disability, depression, anxiety, and pain catastrophizing, and negatively associated with pain-related self-efficacy. The cut-off values for detecting insomnia were estimated at 8 points in the AIS- 8 and 4 points in the AIS-5.

Conclusion: The AIS- 8 and AIS-5 had adequate reliability and validity in chronic pain patients. Keywords: sleep disturbance, AIS, Japanese, insomnia symptom

\section{Introduction}

Sleep disturbance is a prevalent clinical complaint among individuals with chronic pain. ${ }^{1}$ In previous studies, $53 \%-89 \%$ of chronic pain patients had sleep complaints. ${ }^{2-6}$ Sleep disturbance is associated with greater pain intensity, ${ }^{3-6}$ disability, ${ }^{3}$ depression, ${ }^{3,5,6}$ and anxiety ${ }^{6}$ and also with pain-related cognition, such as greater pain catastrophizing and lower pain-related self-efficacy. ${ }^{7,8}$ Therefore, the evaluation and treatment of sleep disturbance are essential components of pain management. ${ }^{9}$

A self-report questionnaire can be used to easily assess difficulties in the sleep of chronic pain patients. The Athens Insomnia Scale (AIS) ${ }^{10,11}$ may be one of the useful measures because it assesses all 3 major insomnia symptoms (difficulties in initiating sleep [DIS], difficulties in maintaining sleep [DMS], and early morning awakening $[\text { EMA] })^{12}$ and important sleep domains (sleep quality and quantity as well as daytime functioning) ${ }^{13}$ Furthermore, the AIS has only 8 items and takes few minutes to complete 
it. The AIS has been translated into many languages ${ }^{10,14-17}$ and has been validated in patients with insomnia, psychiatric disorders, and cancer. ${ }^{10,14-16}$ However, it has not yet been validated in chronic pain patients.

This study aimed to validate the application of the AIS in chronic pain patients. According to the original author, the AIS has 2 versions: AIS- 8 and AIS-5. ${ }^{10}$ The AIS- 8 is the full version, which comprises 8 items related to both nocturnal sleep problems and daytime dysfunction. By contrast, the AIS-5 is the brief version of the AIS and comprises 5 items assessing only nocturnal sleep problems. ${ }^{10,14}$ We hypothesized that: 1) the score of AIS-8 and AIS-5 are positively associated with measures of pain intensity, disability, depression, anxiety, and pain catastrophizing; 2) they have negative correlations with pain-related self-efficacy measures; and 3) patients with insomnia symptoms have higher AIS-8 and AIS-5 scores than patients without insomnia symptoms.

\section{Materials and methods Participants}

This study had a cross-sectional design. Outpatients on their first visit to a pain management center in a university hospital were included as participants. The data of this study were retrospectively extracted from the clinical records between April 2014 and December 2016.

The inclusion criteria were as follows: 1) a history of pain lasting for $\geq 3$ months and 2 ) age $\geq 20$ years. The exclusion criteria were as follows: 1) difficulty in reading and writing Japanese and 2) incomplete interview records of sleep disturbance. For example, if the frequency of DMS was not recorded, the author would be unable to assess the patient's sleep condition.

\section{Measures}

\section{AIS}

The AIS-8 is an 8-item self-report questionnaire that measures the intensity of sleep difficulties ${ }^{10,11}$ according to the International Statistical Classification of Disease and Related Health Problems-10th Revision (ICD-10) diagnostic criteria for insomnia. ${ }^{18}$ Five items assess difficulty in sleep introduction, awakening during the night, early morning awakening, total sleep duration, and overall sleep quality. Three items pertain to the next-day consequences of insomnia (sense of well-being during the day, functioning [physical and mental] during the day, and sleepiness during the day). The AIS-5 is the brief version of the AIS-8 and comprises only 5 items assessing nocturnal sleep problems. Respondents are required to rate positively if they have experienced sleep difficulties at least thrice per week during the last month. Each item is rated on a 4-point numerical rating scale (NRS; where $0=$ no problem at all and $3=$ very serious problem). Total scores range from 0 to 24 in the AIS- 8 and from 0 to 15 in the AIS-5. Higher scores in these AIS measures indicate that responders have severe insomnia symptoms.

The factor structure of the AIS- 8 is different in populations. It has been reported as a 1-factor structure (item 1-8) or a 2-factor structure (item 1-5 and item 6-8) in previous studies. ${ }^{10,14,15,17}$ The factor structure of the AIS-5 is reported as a 1 -factor structure only. ${ }^{14,15}$ Cronbach's $\alpha$ coefficients for the Japanese version of the AIS- 8 and AIS- 5 were 0.88 and 0.85 , respectively. ${ }^{14}$ The cut-off values for detecting insomnia among Japanese outpatients with chronic insomnia were estimated at 6 points in the AIS- 8 and 4 points in the AIS-5. ${ }^{14}$

\section{NRS}

To assess the pain intensity, we determined the average score of 4 items on an 11-point NRS. The NRS ranges from 0 (no pain) to 10 (worst pain imaginable), and the 4 assessed items are as follows: 1) worst pain in the past 24 hours; 2) least pain in the past 24 hours; 3 ) average pain in the past 24 hours; and 4 ) current pain. The average score of these 4 items was used for data analysis. ${ }^{19,20}$

\section{Pain Disability Assessment Scale (PDAS)}

The PDAS is a self-report questionnaire assessing the degree of pain interference. ${ }^{21}$ It comprises 20 items, each of which is rated on a 4-point numerical rating scale. Higher scores (range from 0 to 60) indicate a greater degree of disability. Cronbach's $\alpha$ coefficient of the total PDAS was $0.96 .^{21}$

\section{Hospital Anxiety and Depression Scale (HADS)}

The HADS is a 14-item self-report questionnaire, and each item is rated on a 4-point numerical rating scale. It comprises 2 subscales measuring anxiety (HADS-A) and depression (HADS-D). ${ }^{22}$ Each subscale includes 7 items, and higher scores indicate a greater degree of anxiety or depression. Total scores range from 0 to 42 and each subscale ranges from 0 to 21. Cronbach's $\alpha$ coefficients for the Japanese version of the HADS were 0.77 for HADS-A and 0.79 for HADS-D. ${ }^{23,24}$ The cut-off value was estimated at 11 points in total score and 8 points in HADS-A and HADS-D. ${ }^{25}$

\section{Pain Catastrophizing Scale (PCS)}

The PCS is a self-report questionnaire that assesses the degree of catastrophic thinking regarding pain. It comprises 13 items, each of which is rated on a 5-point numerical rating scale. 
The PCS comprises 3 subscales: rumination, magnification, and helplessness. ${ }^{26}$ Higher scores indicate greater levels of catastrophizing. The total scores range from 0 to 51 . The Japanese version of the PCS has good internal consistency (Cronbach's $a$ coefficients were 0.80 for the rumination subscale, 0.65 for the magnification subscale, 0.81 for the helplessness subscale, and 0.89 for the total score). ${ }^{27}$

\section{Pain Self-Efficacy Questionnaire (PSEQ)}

The PSEQ is a 10-item self-report questionnaire that measures the confidence in performing activities despite pain. ${ }^{28}$ Each item is rated on a 7-point numerical rating scale. Higher scores indicate greater perceived self-efficacy among chronic pain patients. The total scores range from 0 to 60 . The Cronbach's $\alpha$ coefficient for the Japanese version of the PSEQ was $0.94 .^{29}$

\section{Patient's Global Impression of Change (PGIC)}

The PGIC is a self-report questionnaire that measures the change in patients' overall conditions since treatment initiation. ${ }^{30}$ It is a 7-point categorical scale and is scored as follows: 1) very much improved; 2) much improved; 3) minimally improved; 4) no change; 5) minimally worse; 6) much worse; and 7) very much worse. Participants who visited the hospital again were asked to complete this questionnaire. In the present study, we used the PGIC for assessing the test-retest reliabilities of the AIS-8 and AIS-5.

\section{Semi-structured interview for sleep disturbance}

In a semi-structured interview conducted by psychologists, participants were questioned regarding their sleep condition. The items assessed through the interview were wake-up time, bedtime, DIS, DMS, EMA, and history of sleeping pill consumption. DIS was defined as requiring $>30 \mathrm{~min}$ to fall asleep; the presence of DMS and EMA was defined as $>3$ episodes per week. ${ }^{31,32}$ The presence of insomnia was defined as at least 1 positive response to questions regarding DIS, DMS, or EMA. ${ }^{31-34}$

\section{Procedure}

Data were collected as part of routine care at a tertiary pain management center. First, the participants were informed that their questionnaire data and clinical records might be used for clinical and research purposes. If the participants agreed, they were asked to complete questionnaires using an e-tablet with a touch screen in the waiting room. After completing the questionnaires, the patients were consulted by a multidisciplinary team (physician, occupational therapist, physiotherapist, and psychotherapist). During the psychological assessment, the psychologist conducted semi-structured interviews regarding the sleep condition. The interviews were conducted by either of two psychotherapists. Patients who revisited the clinic within 100 days were asked to complete the questionnaires twice. This study was approved by the Institutional Review Board of the Osaka University Hospital (No. 13004-6), and written informed consent was obtained from all participants prior to study inclusion.

\section{Statistical analysis}

All statistical analyses were conducted using R software (version 3.4.1). ${ }^{35}$ Descriptive statistics were calculated to describe the demographic characteristics of the participants. The factor structures of the AIS-8 and AIS-5 were analyzed though confirmatory factor analysis using the package "lavaan" (version 0.5-23). ${ }^{36}$ To determine absolute fit indices, the chi-square goodness-of-fit index $\left(\chi^{2}\right)$, the root mean square error of approximation (RMSEA), and the standardized root mean square residual (SRMR) were used. ${ }^{37}$ An acceptable fitting model was determined as that with a 0.05 threshold using non-significant $\chi^{2}$ tests, ${ }^{37}$ with a RMSEA of $<0.08,{ }^{38}$ and a SRMR of $<0.10 .{ }^{38}$ To assess incremental fit indices, the comparative fit index (CFI) was used. ${ }^{37}$ An acceptable model was determined as that with a CFI of $>0.95 .{ }^{37}$ In addition, the Akaike Information Criterion (AIC) was used as a parsimony fit index. ${ }^{37}$ The best model demonstrated the minimal value of AIC. The $\chi^{2}$ improvement was assessed between the 2 nested models of the AIS- 8 .

Internal consistencies in the AIS-8 and AIS-5 were evaluated using Cronbach's $a$. Test-retest reliability was determined in participants who responded with scores of 3 (minimally improved), 4 (no change), or 5 (minimally worse) on the PGIC at the second visit. To assess test-retest reliability, the interclass correlation coefficient (ICC) was calculated. By using the Pearson correlation coefficients, concurrent validity was evaluated between the AIS and other psychometric measures (the NRS, PDAS, HADS, PCS, and PSEQ) for all participants. Known-group validity was established using the independent $t$-test to compare the mean values of AIS scores between participants with insomnia and those without insomnia defined by semi-structured interview. In the present study, the significance level was set at $p<0.05$. Finally, the cut-off points for the AIS- 8 and AIS-5 were established with receiver-operating characteristic (ROC) curves using the package "pROC" (version 1.10.0). ${ }^{39}$ The optimal cut-off value was determined based on the sensitivity and specificity 
for all participants. The diagnostic accuracy was evaluated by calculating the area under the curve (AUC). Generally, an AUC of 0.9-1.0 represents excellent, 0.8-0.9 represents good, $0.7-0.8$ represents fair, and $0.6-0.7$ represents poor discriminative ability. ${ }^{40}$

\section{Results}

\section{Demographic characteristics of participants}

In total, 144 participants completed the questionnaires and semi-structured interviews. Demographic valuables are shown in Table 1. Eighty-six participants were females $(60.0 \%)$, and the mean age was $53.3(\mathrm{SD}=16.2)$ years. The average pain duration was $53.6(\mathrm{SD}=66.7)$ months. The most frequently reported pain sites were the lower limbs $(68.1 \%)$, followed by upper shoulder and upper limbs (55.6\%), and the lower back (51.4\%). Furthermore, $45.0 \%$ of the participants reported experiencing pain at more than 3 sites. Participants were classified using the recently proposed International Association for the Study of Pain classification. ${ }^{41,42}$ A total of $61.8 \%$ of participants were categorized as Code 1 (chronic primary pain).

Most participants $(88.9 \%)$ had received at least high school education. Marital status was as follows: married (52.8\%), single (25.0\%), divorced (12.5\%), remarried (4.1\%), and widowed (5.6\%). Only $33.4 \%$ of the participants were working. A total of $50.0 \%$ of participants took medication for difficulty sleeping. The prevalence of DIS was $47.9 \%$, of DMS was $75.7 \%$, and of EMA was $18.1 \%$. In total, 131 participants $(91.0 \%)$ exhibited insomnia symptoms. The mean AIS-8 score was 10.8 (SD =5.7), mean AIS-5 score was 6.7 ( $\mathrm{SD}=4.1)$, and mean daytime function (items 6-8) score was $4.1(\mathrm{SD}=2.3)$. The scores for other questionnaires are reported in Table 2 .

\section{Confirmatory factor analysis}

Fit indices revealed that the 1-factor model of the AIS- 8 was a poor fit to the data: $\chi^{2}(20)=121.315, p<0.001, \mathrm{SRMR}=0.082$, RMSEA $=0.188$ (90\% CI: $0.157-0.221), \mathrm{CFI}=0.840$, and $\mathrm{AIC}=2687.137$. Conversely, the 2-factor model of the AIS-8 was an acceptable fit to the data: $\chi^{2}(19)=36.325, p<0.05$, $\mathrm{SRMR}=0.045$, RMSEA $=0.080$ (90\% CI: 0.039-0.119), $\mathrm{CFI}=0.973$, and AIC $=2623.297$. Furthermore, the model fit significantly improved for the 2-factor model $\left(\Delta \chi^{2}[1]=84.99\right.$; $p<0.001)$. Therefore, we adopted the 2-factor structure for the AIS-8 (Figure 1). The factor loading for item 8 was low in the 2-factor model. However, the fit indices were poor if item 8 was removed: $\chi^{2}(13)=32.852, p<0.01, \mathrm{SRMR}=0.051$,
Table I Demographic characteristics of the participants

\begin{tabular}{|c|c|c|}
\hline Demographic characteristics & Frequency (n) & $\%$ \\
\hline Number of participants & 144 & \\
\hline Age (years) & $53.3 \pm 16.2$ & \\
\hline Number of female & 86 & 60.0 \\
\hline Pain durations (months) & $53.6 \pm 66.7$ & \\
\hline \multicolumn{3}{|l|}{ Pain site ${ }^{a}$} \\
\hline Head & 37 & 25.7 \\
\hline Cervical & 40 & 27.8 \\
\hline Upper shoulder and upper limbs & 80 & 55.6 \\
\hline Thoracic & 24 & 16.7 \\
\hline Abdominal & 22 & 15.3 \\
\hline Lower back & 74 & 51.4 \\
\hline Lower limbs & 98 & 68.1 \\
\hline Genital & 6 & 4.2 \\
\hline$>3$ major sites & 65 & 45.0 \\
\hline \multicolumn{3}{|c|}{ Classification of chronic pain according to new IASP classificationa } \\
\hline Code I: chronic primary pain & 89 & 61.8 \\
\hline Code 2: chronic cancer pain & 0 & 0.0 \\
\hline $\begin{array}{l}\text { Code } 3 \text { : chronic postsurgical and } \\
\text { posttraumatic pain }\end{array}$ & 30 & 20.8 \\
\hline Code 4: chronic neuropathic pain & 15 & 10.4 \\
\hline $\begin{array}{l}\text { Code 5: chronic headache and orofacial } \\
\text { pain }\end{array}$ & 4 & 2.8 \\
\hline Code 6: chronic visceral pain & 0 & 0.0 \\
\hline Code 7: chronic musculoskeletal pain & 6 & 4.2 \\
\hline Received at least high school education & 128 & 88.9 \\
\hline \multicolumn{3}{|l|}{ Marital status } \\
\hline Married & 76 & 52.8 \\
\hline Single & 36 & 25.0 \\
\hline Divorced & 18 & 12.5 \\
\hline Remarried & 6 & 4.1 \\
\hline Widowed & 8 & 5.6 \\
\hline \multicolumn{3}{|l|}{ Work status } \\
\hline Working full time & 41 & 28.5 \\
\hline Working part time & 7 & 4.9 \\
\hline Not employed & 52 & 36.1 \\
\hline Retired due to pain & 10 & 6.9 \\
\hline Homemakers & 30 & 20.8 \\
\hline Other & 4 & 2.8 \\
\hline Taking sleep pills & 72 & 50.0 \\
\hline Insomnia symptoms & $|3|$ & 91.0 \\
\hline Difficulty in initiating sleep & 69 & 47.9 \\
\hline Difficulty in maintaining sleep & 109 & 75.7 \\
\hline Early morning awakening & 26 & 18.1 \\
\hline
\end{tabular}

Note: aDuplicate reports of pain site are included.

Abbreviation: IASP, International Association for the Study of Pain.

RMSEA $=0.103$ (90\% CI: 0.060-0.148), and CFI $=0.949$. Thus, the 2-factor model retaining item 8 was finally selected.

For the AIS-5, fit indices revealed that the 1-factor model was marginally acceptable: $\chi^{2}(5)=12.495, p<0.05$, SRMR $=0.045, \mathrm{RMSEA}=0.102$ (90\% CI: 0.031-0.175), $\mathrm{CFI}=0.983$, and $\mathrm{AIC}=1602.073$. To improve the model fit, modification indices suggested the addition of error covariances between items 1 and 5. These items provide a more subjective evaluation of nocturnal sleep problems than items 2-4. Therefore, 
Table 2 Mean, SD, and ranges of scores of psychological measures

\begin{tabular}{|c|c|c|c|c|c|c|}
\hline \multirow[t]{2}{*}{ Measure } & \multicolumn{2}{|c|}{ All patients $(\mathrm{N}=144)$} & \multicolumn{2}{|c|}{$\begin{array}{l}\text { Patients with } \\
\text { insomnia }(\mathrm{N}=|3|)\end{array}$} & \multicolumn{2}{|c|}{$\begin{array}{l}\text { Patients without } \\
\text { insomnia }(\mathrm{N}=13)\end{array}$} \\
\hline & Mean \pm SD & Range & Mean \pm SD & Range & Mean \pm SD & Range \\
\hline AIS-8 (0-24) & $10.8 \pm 5.7$ & $0-24$ & $11.4 \pm 5.6$ & $0-24$ & $5.2 \pm 3.4$ & $0-11$ \\
\hline AIS-5-nocturnal sleep problem (0-15) & $6.7 \pm 4.1$ & $0-15$ & $7.1 \pm 4.1$ & $0-15$ & $2.7 \pm 2.5$ & $0-7$ \\
\hline AIS-daytime dysfunction (0-9) & $4.1 \pm 2.3$ & $0-9$ & $4.3 \pm 2.2$ & $0-9$ & $2.5 \pm 1.9$ & $0-6$ \\
\hline NRS $(0-10)$ & $6.0 \pm 2.0$ & $0.3-10.0$ & $6.2 \pm 1.9$ & $1.8-10.0$ & $4.4 \pm 2.5$ & $0.3-7.8$ \\
\hline PDAS $(0-60)$ & $28.9 \pm \mid 4.8$ & $0-60$ & $29.7 \pm 15.0$ & $0-60$ & $20.5 \pm 10.3$ & $5-34$ \\
\hline HADS-total (0-42) & $19.8 \pm 9.2$ & $1-42$ & $20.4 \pm 9.3$ & $1-42$ & $13.1 \pm 5.3$ & $5-23$ \\
\hline HADS-anxiety (0-2I) & $9.3 \pm 4.8$ & $0-21$ & $9.6 \pm 4.8$ & $0-21$ & $6.2 \pm 3.0$ & $1-10$ \\
\hline HADS-depression (0-2I) & $10.4 \pm 5.2$ & $|-2|$ & $10.8 \pm 5.3$ & $|-2|$ & $6.8 \pm 2.9$ & $3-13$ \\
\hline PCS-total (0-52) & $38.1 \pm 10.0$ & $11-52$ & $38.8 \pm 9.7$ & $\mathrm{II}-52$ & $31.1 \pm 10.9$ & $13-46$ \\
\hline PCS-rumination (0-20) & $17.0 \pm 3.5$ & $0-20$ & $17.1 \pm 3.6$ & $0-20$ & $16.2 \pm 3.2$ & $11-20$ \\
\hline PCS-magnification (0-12) & $7.3 \pm 3.2$ & $0-12$ & $7.6 \pm 3.1$ & $0-12$ & $4.8 \pm 2.8$ & $1-9$ \\
\hline PCS-helplessness (0-20) & $13.7 \pm 4.6$ & $0-20$ & $|4| \pm 4.3$. & $\mathrm{I}-20$ & $10.1 \pm 5.9$ & $0-18$ \\
\hline PSEQ (0-60) & $20.1 \pm 14.1$ & $0-60$ & $19.9 \pm 14.4$ & $0-60$ & $23.0 \pm 11.3$ & $0-42$ \\
\hline
\end{tabular}

Abbreviations: AIS, Athens Insomnia Scale; HADS, hospital anxiety and depression scale; NRS, numerical rating scale; PCS, pain catastrophizing scale; PDAS, pain disability assessment scale; PSEQ, pain self-efficacy questionnaire.

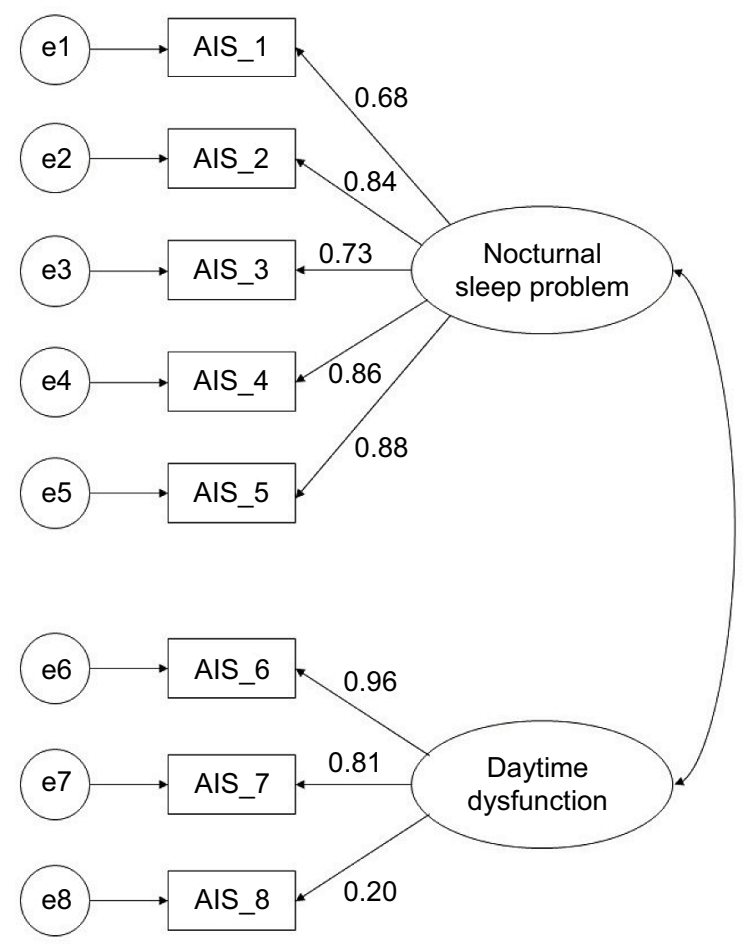

Figure I A 2-factor model of AIS-8.

Abbreviation: AIS, Athens Insomnia Scale.

items 1 and 5 were permitted to covary. With the addition of this error covariance, the model showed a good fit: $\chi^{2}$ (4) $=4.007, p=0.41$, SRMR $=0.013$, RMSEA $=0.004(90 \% \mathrm{CI}$ : $0.000-0.126$ ), $\mathrm{CFI}=1.000$, and $\mathrm{AIC}=1595.526$ (Figure 2).

\section{Internal consistency and test-retest reliability}

Cronbach's $\alpha$ coefficient for the AIS- 8 total score was 0.87 and the AIS-5 was 0.89. In subscales, Cronbach's $a$ coefficient

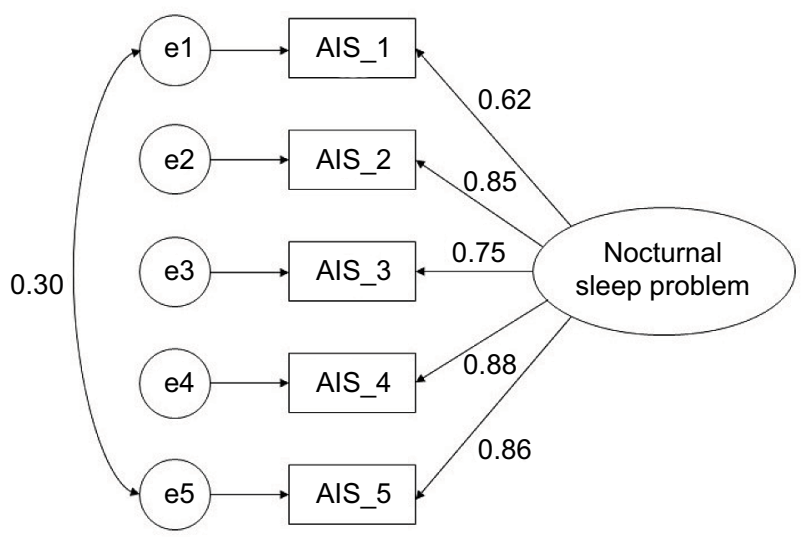

Figure 2 A one-factor model of AIS-5.

Abbreviation: AIS, Athens Insomnia Scale.

was 0.89 for nocturnal sleep problems and 0.66 for daytime dysfunction. Test-retest reliability was analyzed in 43 participants (19 males and 24 females). The mean period between the first and second surveys was $81.4(\mathrm{SD}=19.3$; range $=21-98)$ days. The overall ICC was 0.64 (95\% CI: 0.43 to 0.79 ) for the AIS-8, 0.72 ( $95 \%$ CI: 0.45 to 0.80 ) for the AIS-5 and nocturnal sleep problems, and 0.54 (95\% CI: 0.29 to 0.72 ) for daytime dysfunction.

\section{Concurrent validity}

Correlation analyses revealed that the AIS- 8 had moderate correlations with the PDAS, HADS-A, HADS-D, and PSEQ, and the AIS-5 had moderate correlations with the HADS-A and HADS-D. Moreover, the AIS-8 was weakly associated with the NRS and PCS, and the AIS-5 had weak correlations with the NRS, PDAS, PCS, and PSEQ (Table 3). 


\section{Known-group validity}

Based on the semi-structured interview data, the participants were divided into an insomnia group $(\mathrm{n}=131)$ and noninsomnia group $(\mathrm{n}=13)$. The mean AIS- 8 score was 11.4 ( $\mathrm{SD}=5.6)$ for those with insomnia and $5.2(\mathrm{SD}=3.4)$ for those without insomnia. In addition, the mean AIS-5 score was $7.1(\mathrm{SD}=4.0)$ in participants with insomnia and 2.7 (SD $=2.5$ ) in participants without insomnia. Independent $t$-tests revealed higher AIS scores in participants with insomnia than in those without insomnia in both the AIS- 8 and AIS-5 (AIS$8: t=3.93, d f=142, p<0.001$; AIS-5: $t=3.81, d f=142, p<0.001)$.

\section{Cut-off point}

Based on the ROC analysis, the cut-off value of the AIS- 8 for insomnia was estimated at 8 points, with $72 \%$ sensitivity and $85 \%$ specificity. Furthermore, the cut-off value of the AIS- 5 was estimated at 4 points, with $78 \%$ sensitivity and $70 \%$ specificity (Table 4). The AUC was 0.82 (95\% CI: 0.72 to 0.91 ) for the AIS- 8 and 0.82 (95\% CI: 0.71 to 0.92 ) for the AIS-5. The ROC curves are shown in Figure 3.

\section{Discussion}

The present study aimed to examine the psychometric properties of the AIS- 8 and AIS- 5 in Japanese chronic pain patients.

Table 3 Correlation coefficients between AIS and the other psychological measures

\begin{tabular}{lll}
\hline Measure & AIS-8 $(95 \% \mathrm{Cl})$ & AIS-5 $(95 \% \mathrm{Cl})$ \\
\hline NRS & $0.36(0.2 \mathrm{I}$ to 0.50$)$ & $0.35(0.19$ to 0.48$)$ \\
PDAS & $0.46(0.33$ to 0.58$)$ & $0.37(0.22$ to $0.5 \mathrm{I})$ \\
HADS-anxiety & $0.54(0.4 \mathrm{I}$ to 0.65$)$ & $0.42(0.28$ to 0.55$)$ \\
HADS-depression & $0.64(0.53$ to 0.73$)$ & $0.52(0.39$ to 0.63$)$ \\
PCS-total & $0.36(0.21$ to 0.49$)$ & $0.26(0.10$ to $0.4 \mathrm{I})$ \\
PCS-rumination & $0.23(0.07$ to 0.38$)$ & $0.17(0.01$ to 0.33$)$ \\
PCS-magnification & $0.37(0.22$ to 0.50$)$ & $0.27(0.1 \mathrm{I}$ to 0.42$)$ \\
PCS-helplessness & $0.35(0.19$ to 0.48$)$ & $0.24(0.08$ to 0.39$)$ \\
PSEQ & $-0.47(-0.59$ to -0.33$)$ & $-0.35(-0.48$ to -0.19$)$ \\
\hline
\end{tabular}

Abbreviations: AIS, Athens Insomnia Scale; HADS, hospital anxiety and depression scale; NRS, numerical rating scale; PCS, pain catastrophizing scale; PDAS, pain disability assessment scale; PSEQ, pain self-efficacy questionnaire.
The 2-factor model of the AIS-8 and 1-factor model of the AIS- 5 showed a good fit. The AIS- 8 and AIS- 5 had adequate reliability. All the hypotheses were supported. Thus, the AIS was confirmed to exhibit concurrent validity and known-group validity. According to the ROC analysis, the cut-off value of the AIS- 8 was estimated at 8 points and AIS- 5 was estimated at 4 points. Our data illustrated that the AIS is a measure with good reliability and validity in chronic pain patients.

A 2-factor model of the AIS-8 showed better fit indices than a 1-factor in this study. The factor structure of the AIS- 8 in the present study is similar to that in a prior study conducted at a sleep clinic recruiting individuals with insomnia in Japan. ${ }^{14}$ According to the developers of the original version of the AIS, items 1-5 are based on the criterion A for the diagnosis of insomnia according to ICD-10 and items 6-8 are based on the criterion C of the ICD-10. ${ }^{10,18}$ Based on this notion, it is reasonable that the 2 -factor model of the AIS- 8 was an acceptable fit to the data.

In this study, the factor loading for sleepiness during the day (item 8) was lower than that in previous studies $(0.45-0.63) .^{10,14,15}$ Although the reason is uncertain, some explanations are available. First, chronic pain patients might concentrate on their pain rather than their sleepiness in the daytime and do not mind daytime sleepiness. Second, because $66.6 \%$ of participants were currently not at work, they might be less bothered by their daytime sleepiness. Further studies are needed to examine the factor loading for item 8 in chronic pain patients.

We confirmed that the AIS- 8 and AIS- 5 have high internal consistency. The Cronbach's $\alpha$ value was similar to that of the Japanese version for an insomnia sample (AIS-8: $\alpha=0.88$ and AIS-5: $\alpha=0.85) \cdot{ }^{14}$ However, the overall ICCs of the AIS-8 and AIS-5 were low (AIS-8: ICC $=0.64$ and AIS-5: ICC $=0.72$ ). The ICC was 0.89 for AIS- 8 and 0.88 for AIS- 5 in the original version. ${ }^{10}$ This difference might have occurred due to the longer interval in the present study.

The score of AIS was positively associated with pain intensity, disability, depression, anxiety, and pain

Table 4 Sensitivity and specificity values of the AIS

\begin{tabular}{lllll}
\hline Measure & Cut-off score & Sensitivity (\%) & Specificity (\%) & AUC (95\% Cl) \\
\hline AIS-8 & 7 & 75 & 69 & $0.82(0.72$ to 0.91$)$ \\
& $8^{a}$ & $\mathbf{7 2}^{\mathrm{a}}$ & $\mathbf{8 5}^{\mathrm{a}}$ & \\
& 9 & 66 & 85 & $0.82(0.71$ to 0.92$)$ \\
AIS-5 & 3 & 83 & 46 & \\
& $\mathbf{4}^{\mathrm{a}}$ & $\mathbf{7 8}^{\mathrm{a}}$ & $\mathbf{7 0}^{\mathrm{a}}$ & \\
& 5 & 69 & 76 & \\
\hline
\end{tabular}

Note: aptimal cutoff score values are shown in bold.

Abbreviations: AIS, Athens Insomnia Scale; AUC, area under the curve. 
A

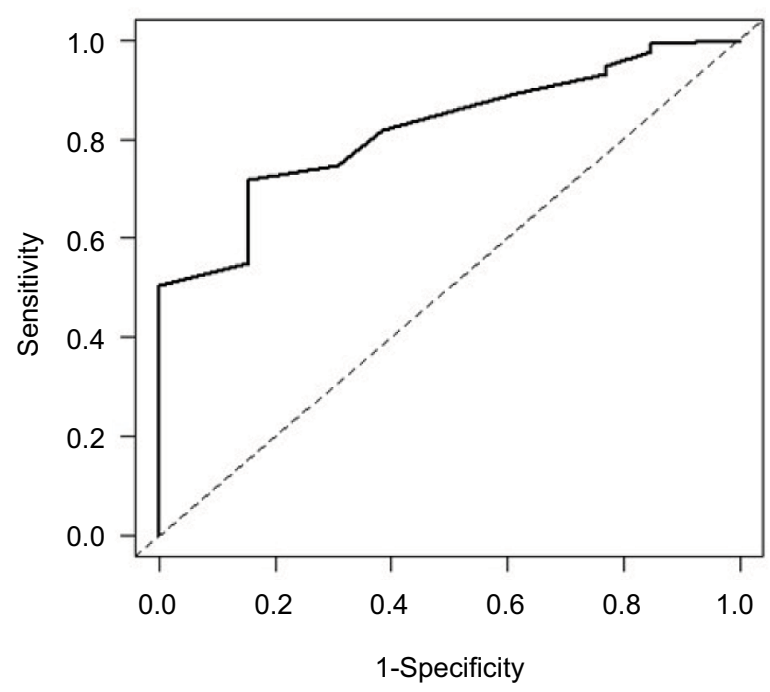

Figure 3 ROC curve of the AIS- 8 and AIS-5.

Notes: (A) ROC curve of the AIS-8. (B) ROC curve of the AIS-5.

Abbreviations: AIS, Athens Insomnia Scale; ROC, receiver-operating characteristic.

catastrophizing, and negatively associated with self-efficacy in the current study. In previous studies, sleep disturbance was associated with greater pain intensity, ${ }^{3-6}$ disability, ${ }^{3}$ depression, ${ }^{3,5,6}$ anxiety, ${ }^{6}$ and pain catastrophizing. ${ }^{7}$ In addition, poor sleep quality was associated with worse levels of self-efficacy in patients with fibromyalgia. ${ }^{8}$ Therefore, the correlation between the AIS and other psychometric questionnaires is applicable. Concurrent validity was confirmed for the AIS.

The cut-off value of the AIS- 8 was higher than that of the original and Japanese versions ( 6 points for both) for an insomnia sample. ${ }^{10,14}$ By contrast, the cut-off value of the AIS-5 was the same as that of the Japanese version for patients with insomnia. ${ }^{14}$ The cut-off value of only the AIS- 8 was higher than that of the original version because the current participants with chronic pain might have rated their daytime dysfunction (items 6-8) more positively than participants with primary insomnia. Actually, the mean daytime dysfunction score in this study was higher than that of the primary insomnia group (3.65; $\mathrm{SD}=1.95) .{ }^{14}$ According to Dueñas's review, chronic pain severely affects the patients' daily activities and quality of life. ${ }^{43}$ Many chronic pain patients are less able to exercise, walk, work outside home, attend social activities, and do household chores. ${ }^{2}$ Hence, because of pain, chronic pain patients with insomnia would feel more daytime dysfunction than patients with primary insomnia.

\section{Limitations}

This study has some limitations. First, the number of patients without insomnia is low $(\mathrm{N}=13)$, and the selection bias might

\section{B}

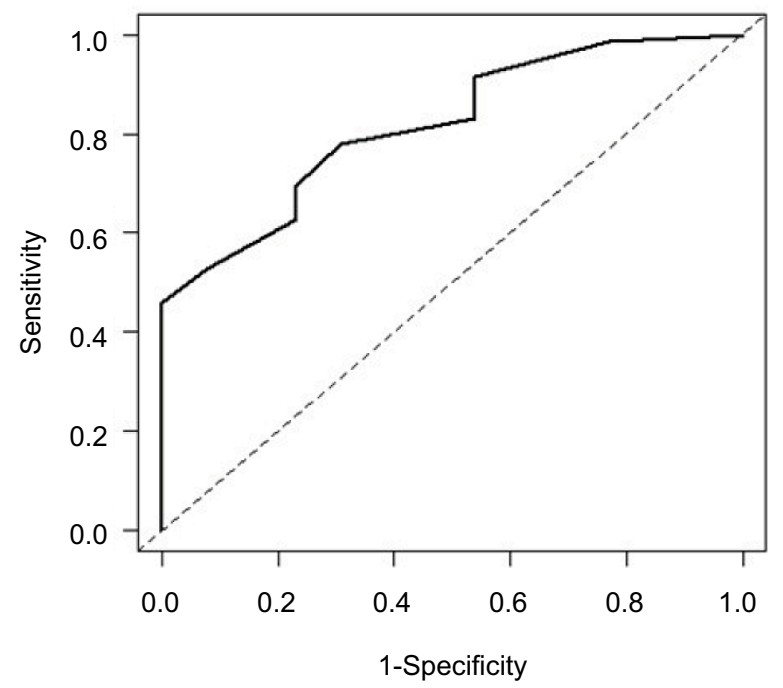

have occurred in this study. The low number of non-insomnia patients may be due to the participants in this study being recruited in a tertiary care setting, in which many patients may experience severe pain and insomnia. In previous studies, $92.9 \%$ chronic pain patients reported difficulty with sleep in tertiary setting, ${ }^{4}$ while $74.9 \%$ patients with chronic pain reported sleep disturbance in primary care settings. ${ }^{44}$ The patients in current study would experience more insomnia than those in primary or secondary care settings.

Second, this study did not apply any other insomnia scales (such as the Pittsburgh Sleep Quality Index ${ }^{45}$ and Insomnia Severity Index ${ }^{46}$ ) or objective measures of sleep (e.g., actigraphy and polysomnography). In previous studies, other insomnia scales and Actiwatch parameters have been measured to confirm the validity of the AIS. ${ }^{14,16,17}$ Furthermore, the semi-structured interview for insomnia was brief. The diagnostic criteria of Diagnostic and Statistical Manual of Mental Disorders-IV-Text Revision were used to divide participants into insomnia and non-insomnia groups in previous studies. ${ }^{16,17}$

In spite of these limitations, we confirmed good psychometric properties of the AIS in chronic pain patients. The AIS is widely used for assessing the risk of insomnia, ${ }^{12}$ because it is short and assesses important sleep domain. ${ }^{12,13}$ The AIS is expected to assess insomnia symptom of chronic pain patients not only in research settings, but also in clinical settings.

\section{Conclusion}

This study demonstrated that the AIS-8 and AIS-5 have excellent reliability and validity in chronic pain patients. 
The cut-off value for screening insomnia was estimated at 8 points in the AIS- 8 and 4 points in the AIS-5. The AIS has high utility because it assesses all the 3 major insomnia symptoms and takes only a few minutes to complete. The AIS is useful for assessing insomnia symptoms of chronic pain patients in research and clinical practice.

\section{Author contributions}

All authors contributed toward data analysis, drafting and revising the paper and agree to be accountable for all aspects of the work.

\section{Disclosure}

The author reports no conflicts of interest in this work.

\section{References}

1. Menefee LA, Cohen MJM, Anderson WR, Doghramji K, Frank ED, Lee $\mathrm{H}$. Sleep disturbance and nonmalignant chronic pain: a comprehensive review of the literature. Pain Med. 2000;1(2):156-172.

2. Breivik H, Collett B, Ventafridda V, Cohen R, Gallacher D. Survey of chronic pain in Europe: prevalence, impact on daily life, and treatment. Eur J Pain. 2006;10(4):287-333.

3. McCracken LM, Iverson GL. Disrupted sleep patterns and daily functioning in patients with chronic pain. Pain Res Manag. 2002;7(2):75-79.

4. McCracken LM, Williams JL, Tang NKY. Psychological flexibility may reduce insomnia in persons with chronic pain: a preliminary retrospective study. Pain Med. 2011;12(6):904-912.

5. Smith MT, Perlis ML, Smith MS, Giles DE, Carmody TP. Sleep quality and presleep arousal in chronic pain. J Behav Med. 2000;23(1):1-13.

6. Tang NKY, Wright KJ, Salkovskis PM. Prevalence and correlates of clinical insomnia co-occurring with chronic pain. J Sleep Res. 2007;16(1):85-95.

7. Roberts MB, Drummond PD. Sleep problems are associated with chronic pain over and above mutual associations with depression and catastrophizing. Clin J Pain. 2016;32 (9):792-799.

8. Miró E, Martínez MP, Sánchez AI, Prados G, Medina A. When is pain related to emotional distress and daily functioning in fibromyalgia syndrome? The mediating roles of self-efficacy and sleep quality. $\mathrm{Br} J$ Health Psychol. 2011;16(4):799-814.

9. Nicholas M, Keefe FJ, Lautenbacher S. Pain, anxiety, depression, and psychological evaluation and treatment. In: Claudia LS, Mark SW, Steven PC, Michaela K, editors. Pain 2016 Refresher Courses, 16th World Congress on Pain. Washington, DC: IASP Press; 2016:379-398.

10. Soldatos CR, Dikeos DG, Paparrigopoulos TJ. Athens Insomnia Scale: validation of an instrument based on ICD-10 criteria. J Psychosom Res. 2000;48(6):555-560.

11. Soldatos CR, Dikeos DG, Paparrigopoulos TJ. The diagnostic validity of the Athens Insomnia Scale. J Psychosom Res. 2003;55(3):263-267.

12. Chiu HY, Chang LY, Hsieh YJ, Tsai PS. A meta-analysis of diagnostic accuracy of three screening tools for insomnia. J Psychosom Res. 2016;87:85-92.

13. Wells GA, Li T, Kirwan JR, et al. Assessing quality of sleep in patients with rheumatoid arthritis. J Rheumatol. 2009;36(9):2077-2086.

14. Okajima I, Nakajima S, Kobayashi M, Inoue Y. Development and validation of the Japanese version of the Athens Insomnia Scale. Psychiatry Clin Neurosci. 2013;67(6):420-425.

15. Gómez-Benito J, Ruiz C, Guilera G. A Spanish version of the Athens Insomnia Scale. Qual Life Res. 2011;20(6):931-937.

16. Sun JL, Chiou JF, Lin CC. Validation of the Taiwanese version of the Athens Insomnia Scale and assessment of insomnia in Taiwanese cancer patients. J Pain Symptom Manage. 2011;41(5):904-914.
17. Jeong HS, Jeon Y, Ma J, et al. Validation of the Athens Insomnia Scale for screening insomnia in South Korean firefighters and rescue workers. Qual Life Res. 2015;24(10):2391-2395.

18. World Health Organization. The ICD-10 Classification of Mental and Behavioural Disorders: Clinical Descriptions and Diagnostic Guidelines. Geneva: World Health Organization; 1992.

19. Serlin RC, Mendoza TR, Nakamura Y, Edwards KR, Cleeland CS. When is cancer pain mild, moderate or severe? Grading pain severity by its interference with function. Pain. 1995;61(2):277-284.

20. Jensen MP, Turner JA, Romano JM, Fisher LD. Comparative reliability and validity of chronic pain intensity measures. Pain. 1999;83(2):157-162.

21. Yamashiro K, Arimura T, Iwaki R, Jensen MP, Kubo C, Hosoi M. A multidimensional measure of pain interference: reliability and validity of the Pain Disability Assessment Scale. Clin J Pain. 2011;27(4): 338-343.

22. Zigmond AS, Snaith RP. The hospital anxiety and depression scale. Acta Psychiatr Scand. 1983;67(6):361-370.

23. Kitamura T. Hospital anxiety and depression scale (in Japanese). Seisinka Sindangaku. 1993;4(3):371-372.

24. Kugaya A, Akechi T, Okuyama T, Okamura H, Uchitomi Y. Screening for psychological distress in Japanese cancer patients. Jap J Clin Oncol. 1998;28(5):333-338.

25. Bjelland I, Dahl AA, Haug TT, Neckelmann D. The validity of the Hospital Anxiety and Depression Scale. An updated literature review. J Psychosom Res. 2002;52(2):69-77.

26. Sullivan MJL, Bishop SR, Pivik J. The pain catastrophizing scale: development and validation. Psychol Assess. 1995;7(4):524-532.

27. Matsuoka H, Sakano Y. Assessment of cognitive aspect of pain: development, reliability, and validation of Japanese version of pain catastrophizing scale (in Japanese). J Psychosom Med. 2007;47(2): 95-102.

28. Nicholas MK. The pain self-efficacy questionnaire: taking pain into account. Eur J Pain. 2007;11(2):153-163.

29. Adachi T, Nakae A, Maruo T, et al. Validation of the Japanese version of the pain self-efficacy questionnaire in Japanese patients with chronic pain. Pain Med. 2014;15(8):1405-1417.

30. Farrar JT, Young JP Jr, LaMoreaux L, Werth JL, Poole RM. Clinical importance of changes in chronic pain intensity measured on an 11-point numerical pain rating scale. Pain. 2001;94(2):149-158.

31. Nakata A, Ikeda T, Takahashi M, et al. Sleep-related risk of occupational injuries in Japanese small and medium-scale enterprises. Ind Health. 2005;43(1):89-97.

32. Takahashi M, Iwakiri K, Sotoyama M, et al. Work schedule differences in sleep ploblems of nursing home caregivers. Appl Ergon. 2008;39(5):597-604.

33. Kaneita Y, Oshida T, OsakiY, et al. Insomnia among Japanese adolescents: a nationwide representative survey. Sleep. 2006;29(12):1543-1550.

34. Nakata A, Haratani T, Takahashi M, et al. Job stress, social support, and prevalence of insomnia in a population of Japanese daytime workers. Soc Sci Med. 2004;59(8):1719-1730.

35. R Core Team. R: A language and environment for statistical computing. Vienna, Austria: R Foundation for Statistical Computing; 2017. Available from: https://www.R-project.org/. Accessed October 04, 2017.

36. Rosseel Y. lavaan: an R package for structural equation modeling. J Stat Softw. 2012;48(2):1-36. Available from: http://www.jstatsoft.org/v48/ i02/. Accessed October 04, 2017.

37. Hooper D, Coughlan J, Mulle MR. Structural equation modelling: guidelines for determining model fit. EJBRM. 2008;6(1): $53-60$.

38. Schermelleh-Engel K, Moosbrugger H, Muller H. Evaluating the fit of structural equation models: tests of significance and descriptive goodness-of fit measures. MPR Online. 2003;8(2):23-74.

39. Robin X, Turck N, Hainard A, et al. pROC: an open-source package for $\mathrm{R}$ and $\mathrm{S}+$ to analyze and compare ROC curves. BMC Bioinformatics. 2011;12:73. Available from: http://www.biomedcentral.com/14712105/12/77/. Accessed October 04, 2017. 
40. Swets JA. Measuring the accuracy of diagnostic systems. Science. 1988;240(4857):1285-1293.

41. Treede RD, Reif W, Barke A, et al. A classification of chronic pain for ICD-11. Pain. 2015;156(6):1003-1007.

42. Takura $\mathrm{T}$, Shibata $\mathrm{M}$, Inoue $\mathrm{S}$, et al. Socioeconomic value of intervention for chronic pain. J Anesth. 2016;30(4):553-561.

43. Dueñas M, Ojeda B, Salazar A, Mico JA, Failde I. A review of chronic pain impact on patients, their social environment and the health care system. J Pain Res. 2016;28(9):457-467.
44. Salazar A, Dueñas M, Mico JA, et al. Undiagnosed mood disorders and sleep disturbances in primary care patients with chronic musculoskeletal pain. Pain Med. 2013;14(9):1416-1425.

45. Buysse DJ, Reynolds CF 3rd, Monk TH, Berman SR, Kupfer DJ. The Pittsburgh Sleep Quality Index: a new instrument for psychiatric practice and research. Psychiatry Res. 1989;28(2):193-213.

46. Bastien CH, Vallières A, Morin CM. Validation of the Insomnia Severity Index as an outcome measure for insomnia research. Sleep Med. 2001;2(4):297-307.

\section{Publish your work in this journal}

The Journal of Pain Research is an international, peer reviewed, open access, online journal that welcomes laboratory and clinical findings in the fields of pain research and the prevention and management of pain. Original research, reviews, symposium reports, hypothesis formation and commentaries are all considered for publication.

\section{Dovepress}

The manuscript management system is completely online and includes a very quick and fair peer-review system, which is all easy to use. Visit http://www.dovepress.com/testimonials.php to read real quotes from published authors. 